\title{
INTERFEROMETRIC OBSERVATION \\ OF CYGNUS-A DISCRETE RADIOSOURCE SCINTILLATIONS AT IRKUTSK INCOHERENT SCATTER RADAR
}

\author{
M.V. Globa, R.V. Vasilyev, D.S. Kushnaryov, A.V. Medvedev
}

Institute of Solar-Terrestrial Physics SB RAS,

Irkutsk,Russia,globa@iszf.irk.ru

We propose a new method for analyzing data from the Irkutsk Incoherent Scatter Radar. The method allows us to accomplish interferometric observation of discrete cosmic radio source characteristics. In this study, we analyze ionospheric scintillations of the radio source Cygnus-A. Observations were made in 2013 during regular radar sessions within 5-15 days for different seasons; the effective time of observation was 15-30 minutes per day. For interferometric analysis, the properties of correlation (coherence) coefficient of two independent recording channels were used. The statistical analysis of data from the independent channels allows us to construct two-dimensional histograms of radio source brightness distribution with a period of $18 \mathrm{~s}$ and to determine parameters (the maximum position and the histogram width) representing position and angular size of the radio source for each histogram. It is shown that the change of statistical characteristics does not correlate with fluctuations in power (scintillations) of the signal induced by radio wave propagation through ionospheric irregularities.

Keywords: ionospheric irregularities, discrete radio source scintillations.

\section{INTRODUCTION}

Radio signal scintillation in Earth's ionosphere is a quite thoroughly studied [Kung Chie Yeh, ChaoHan Liu, 1982] phenomenon known since the mid-twentieth century [Little, Maxwell, 1951]. The main areas of human activity significantly affected by ionospheric scintillations are satellite communications and radio astronomy. At present there are a number of models that can describe and to some extent predict ionospheric scintillation dynamics on the planetary scale [Priyadarshi, 2015]. In some cases, studying properties of scintillations makes it possible to determine characteristics of ionospheric irregularities [Bezrodny et al., 2010]. The Irkutsk Incoherent Scatter Radar (IISR) operating in passive mode can also observe scintillations of radio waves from discrete cosmic radio sources (DCR) in the ionosphere [Vasilyev et al., 2013]. The IISR registration system provides a means for interferometric [Lebedev et al., 2013] observation of the ionosphere and near-Earth objects. It can also be used to examine DCR and their characteristics. 


\section{INTERFEROMETRY OF IONOSPHERIC SCINTILLATIONS}

Propagation of meter radio waves in the ionosphere generally has a detrimental effect on radio telescope-interferometers. Refraction effects caused by signal propagation in an inhomogeneous medium hamper accurate determination of an emission source and its size [Spoelstra, Kelder, 1984]. Variations of ionospheric characteristics are different: seasonal and diurnal variations of electron density, its evolution with height, as well as wave phenomena (traveling ionospheric disturbances) with characteristic periods of about several hours and sizes from tens to thousands of kilometers. These relatively smooth variations of ionospheric parameters have an effect on radio interferometers with aerial spacing of around several kilometers and more. They can be taken into account by the use of either existing ionospheric models [Sukumar, 1987] or auxiliary ionospheric observation. Ionospheric irregularities about one kilometer in size being close to the Fresnel zone size for meter waves at ionospheric heights cause diffraction effects manifesting themselves as DCR scintillations. They are recorded by an interferometer during DCR observation and can be different - depending on the ratio of sizes of irregularities to aerial spacing we can perceive the difference between amplitude and phase scintillations at the output of the correlator. The size of the aerial spacing being much smaller than that of irregularity, radio signal variations in different antennas of the interferometer are synchronous and there are variations in the signal-to-noise ratio. This is a manifestation of so-called amplitude scintillations. In this case, the relative signal phase varies slowly in response to DCR movement along the celestial sphere due to daily rotation. If the aerial spacing is enlarged to sizes comparable to those of ionospheric irregularities or exceeding them, phase delays of signals in antennas of the interferometer caused by radio wave propagation through areas with diverse electron densities become different. This results in a summation of signals with different delays in the correlator; and the signal variation at the output of the correlator is already conditioned both by a variation of the signal-to-noise ratio and by amplification and attenuation of the output signal due to inphase and anti-phase summation. This is a manifestation of phase scintillations. Here the smooth phase variation of the signal from DCR due to the Earth rotation is overlapped by fast phase variations which in the end influence the observed location and size of DCR. This issue has been studied in more detail in [Spoelstra, Yang Yi-Pei, 1995]. The authors showed that weakening of amplitude scintillations and intensification of phase scintillations depend on the size of aerial spacing.

\section{THE IRKUTSK INCOHERENT SCATTER RADAR AS A RADIOINTERFEROMETER}

The IISR antenna system designed for frequency scanning of a celestial-sphere area $20^{\circ} \times 30^{\circ}$ in size represents two independent adjoining combined sectoral horns (half-horns). Linear sizes of this antenna system are $250 \times 20 \times 12 \mathrm{~m}$; the total mouth of the half-horns $(250 \times 12 \mathrm{~m})$ is $3000 \mathrm{~m}^{2}$. Slot radiators of feeding waveguides at the base of each the half-horns constitute a linear antenna array ensuring a $0.5^{\circ}$ antenna diagram (AD) width in the plane of scan; walls of the half-horns provide a $20^{\circ} \mathrm{AD}$ width in the plane perpendicular to the plane of scan. The effective area of antenna aperture is $\sim 1000 \mathrm{~m}^{2}$. The IISR frequency range is $154-162 \mathrm{MHz}$. This corresponds to a wavelength of about two meters. The radar AD shape as well as exact coefficients of scanning equation can be obtained from DCR observation. 
Simultaneous observation of DCR by the two half-horns also allows us to determine characteristics of the interferometer they form. The slope of the characteristic linking the DCR movement along the celestial sphere with the signal phase difference in the half-horns is 16 electric degrees per one degree of the celestial sphere [Lebedev et al., 2006]. The distance between antenna phase centers in this case is about 5 $\mathrm{m}$. The aerial spacing based on the IISR antenna system is small, and therefore the width of the main lobe is about $11^{\circ}$. The $\mathrm{AD}$ width of both the half-horns in the plane with phase centers is around $20^{\circ}$. Thus, the main lobe of the interferometer is fully within the antenna system's AD.

Signals in the half-horns are registered by a dedicated system that allows a signal, received by each of the horns, to be stored as a series of complex numbers in long-term storage. The system includes: 1) an analog amplifying-heterodyne subsystem that amplifies a received signal and modulates to a frequency range appropriate for digital recorders; 2) a hexadecimal D-A converter that registers a signal at the output of the analog subsystem with a digitization frequency of $1 \mathrm{MHz}$; and 3) a signaling processor resolving a D-A converter result into complex components. The system registers signals at intervals of 8 $\mu$ s (time realizations) with a period of about $40 \mu$ s. System operation and final data representation are performed by a controller computer. The observation results obtained by the computer are sent to the IISR local area network and are saved on hard disks of a server computer for further processing and storage.

The complex registration of signals is convenient for correlation processing. There is no need to develop an auxiliary device (correlator) including into the measuring system; an opportunity exists to vary integration time in further processing, etc. In the correlation data analysis, we use a cross-correlation coefficient of signals in the horns of the antenna system

$$
\eta=\frac{\left\langle A B^{*}\right\rangle}{\left\langle A A^{*}\right\rangle\left\langle B B^{*}\right\rangle},
$$

where $A$ and $B$ are signals from independent horns (interferometer antennas); the asterisk denotes complex conjugation operation; the angle brackets, time averaging. It is easy to show that the argument of the resulting value represents the source position relative to phase centers; the modulus, the signal-tonoise ratio (see Appendix). The coherence ratio is traditionally considered as a statistical characteristic; and formally we can derive for it not only a mean value, but also higher-order moments reflecting changes in the shape of the observed DCR. However, the noise component in a signal may make statistical moments undergo variations unrelated to ionospheric variations of DCR size and location. That is why we have come to the decision to examine statistical distributions (histograms), instead of average characteristics, and, by analyzing shapes of these distributions, draw conclusions about the observed location of the source on the celestial sphere and its size.

\section{TWO-DIMENSIONAL HISTOGRAM OF THE RADIO SOURCE CYGNUS-A AND ITS CHARACTERISTICS}

The radio galaxy Cygnus-A is one of the brightest radio sources in the northern celestial hemisphere. It passes through the IISR scanned area every day; therefore it is highly suitable for data analysis. Active observations at IISR are made using a fixed frequency. This corresponds to the AD tilt in a certain 
direction in the scanned area. During active observations, we can choose an instant of time at which the DCR Cygnus-A is passing through AD, and utilize the data acquired at this instant to study the DCR. The data represent time realizations of a received signal which comprise both the sounding signal, scattered in the ionosphere, and background cosmic radio emission. Consequently, we should neglect the beginning of the realization with significant sounding signal and examine only its final part from a distance of $\sim 600$ $\mathrm{km}$. A shift in the frequency of the received radio emission implies a displacement of the source along the north-south plane; and a change in the phase difference between the two half-horns, a displacement along the west-east plane. Determining DCR coordinates in these directions requires analyzing the frequency spectrum of the received radio signal and argument of radio signal correlation coefficient in half-horns (1). During data processing, we performed the Fourier transform for each realization and then calculated the argument of correlation coefficient between the half-horns for each frequency component:

$$
\arg \left(\eta_{i}\right)=\arg \left(a_{i} b_{i}\right)
$$

where $i$ is a frequency component number; $a, b$ are spectral readings of signals from the half-horns. These values were accumulated in a two-dimensional histogram over a period of $18 \mathrm{~s}$. Over the same period, we amassed correlation coefficient values:

$$
H_{i}=\sum_{k} \frac{a_{i k} b_{i k}^{*}}{\sqrt{a_{i k} a_{i k}^{*} b_{i k} b_{i k}^{*}}}
$$

where $k$ marks time readings; $i$, frequency readings.

Each value of the accumulated two-dimensional histogram was multiplied by a modulus of relevant correlation coefficient for normalization. Thus, we formed frequency and phase distribution of DCR signal power. This distribution could of course be considered as an image of the radio source, but such an image is deficient since the power distribution along one of the coordinates (phase) was obtained from the interferometer with one base and in fact it represents the behavior of an effective center of gravity of a full image along this coordinate. Power distribution along the other coordinate (frequency) is a convolution of the image with AD. For convenience and brevity, this two-dimensional histogram of frequency and phase distribution of DCR power will be termed radio source image. Figure 1 presents three images captured at subsequent points in time.

The source passes through $\mathrm{AD}$ for 10-15 min. To analyze the DCR movement along the radar $\mathrm{AD}$, we calculate the maximum position and the mean brightness for each image (Figure 2, $a, b$ ). In the absence of DCR, the maximum position varies in a random manner, whereas the mean value remains relatively constant. In the presence of the source, the mean value varies slowly and does not quite correctly represent the source movement along the scanned area. Consequently, we can conclude that to observe the source position it is better to keep track of the maximum distribution. 

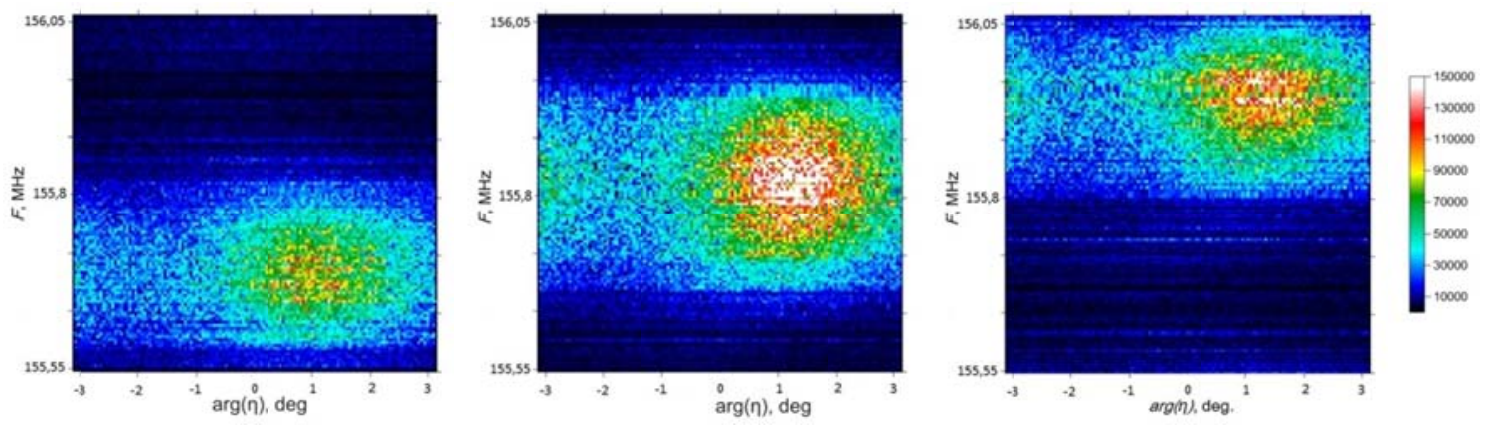

Figure 1. Radio source images plotted during the accumulation period of $18 \mathrm{~s}$
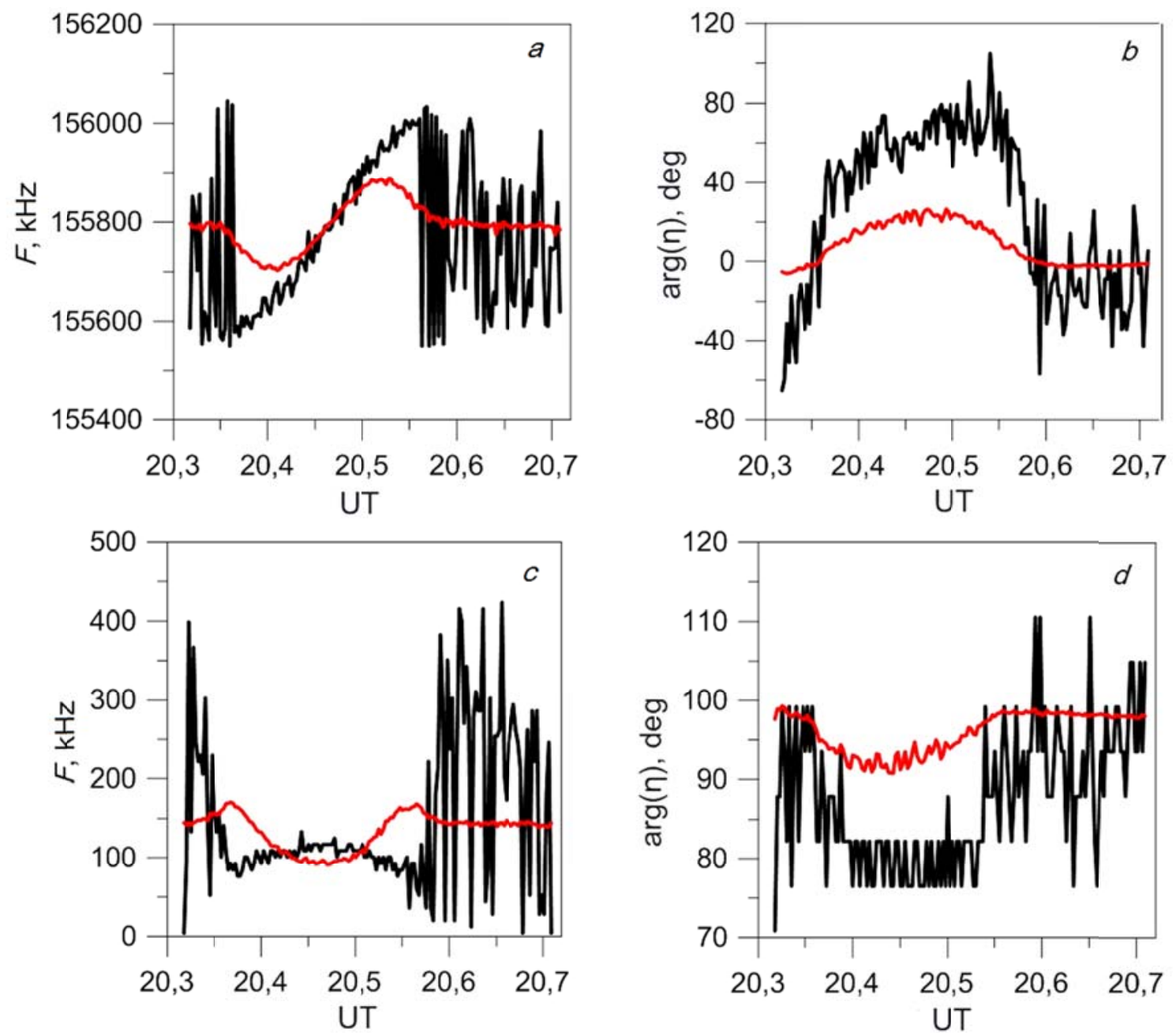

Figure 2. Change in the position of the maximum (black line) and average (red line) distributions for frequency (a) and phase $(b)$; change in the standard deviation (red line) and width of the histogram (black line) for frequency $(c)$ and phase $(d)$ on June 28,2013

To analyze the DCR image width, we compute the standard deviation and width of the histogram at the level of half amplitude (Figure 2,c,d). It is apparent that for frequency (Figure 2,c) the standard deviation also slowly varies in the presence of the source and inaccurately represents the change in the image width. 


\section{VARIATIONS IN CHARACTERISTICS OF THE RADIO SOURCE CYGNUS-A UNDER CONDITIONS OF SCINTILLATIONS AND ALTERNATION OF SEASONS}

At normal IISR functioning, radio signals are being registered continuously for sevieral days and nights. $\mathrm{AD}$ is incompletely scanned. We generally select two fixed directions that are cyclically within the radar view.
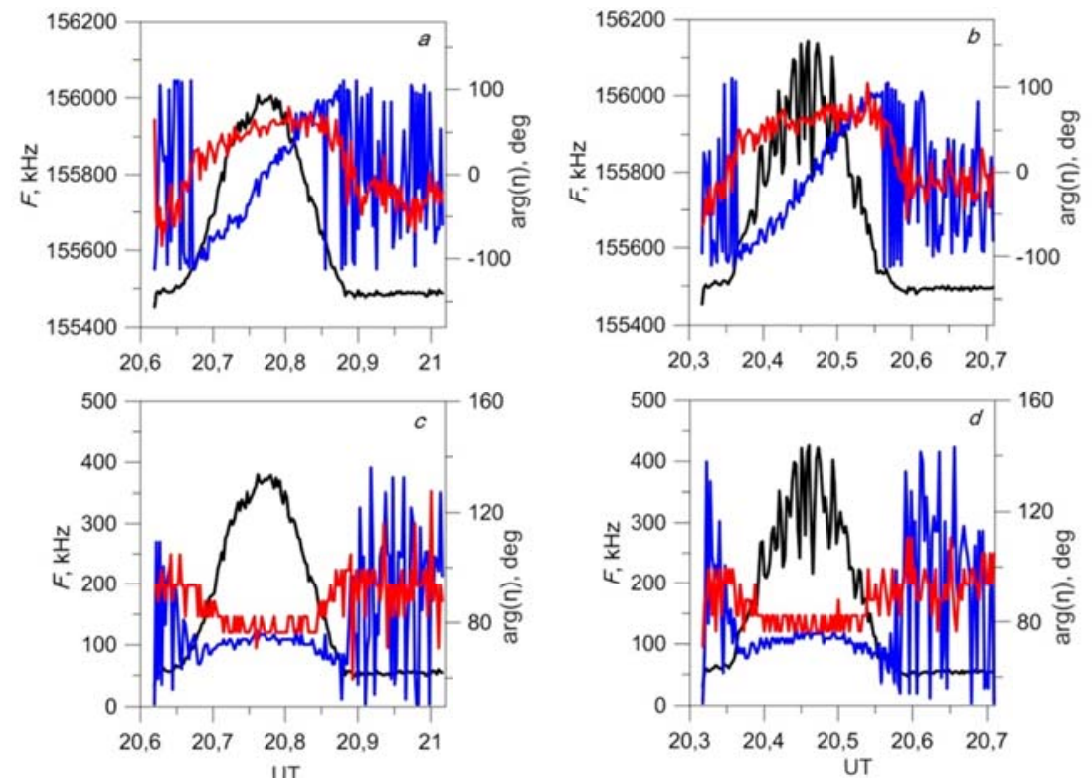

Figure 3. Variations in power (black line) and main characteristics of the DCR image for the broadband channel (500 kHz). On panels $a, b$, the blue curve depicts the change in the frequency distribution maximum position; the red one, the change in the phase distribution maximum position; on panels $c, d$, the blue curve shows the width change at a half-height in frequency; the red one, that in phase on June $23(a, c)$ and June $28(b, d), 2013$
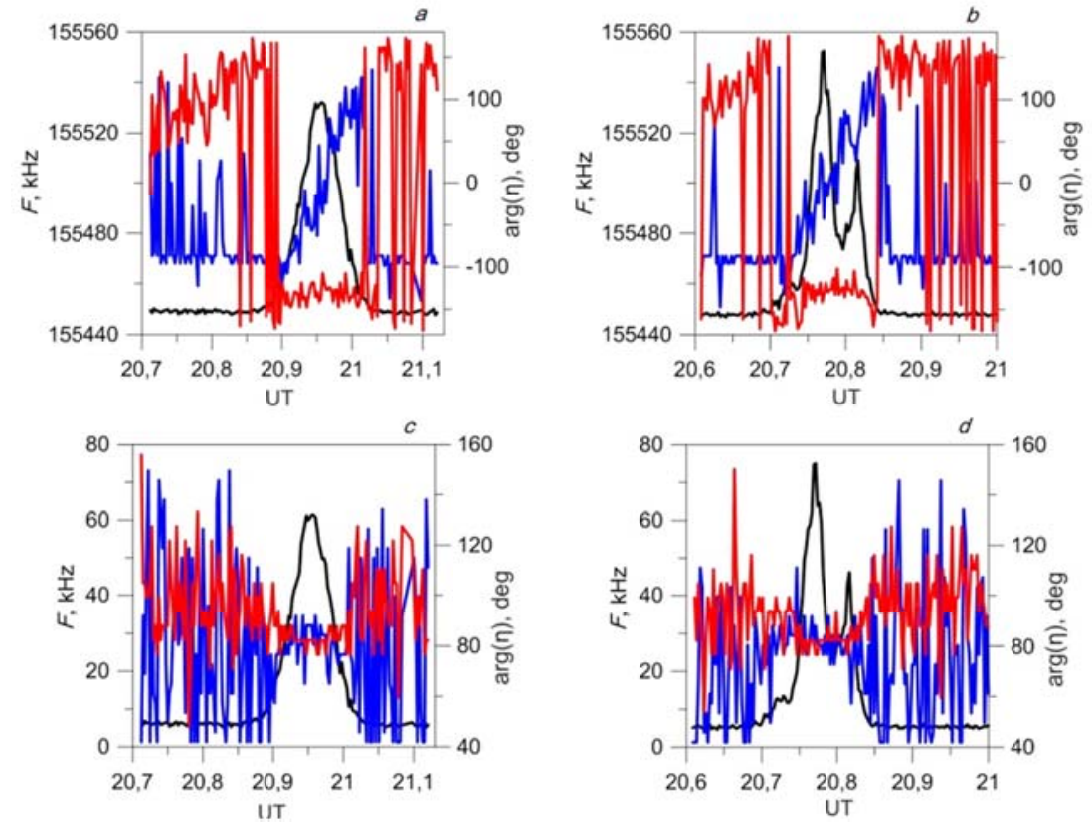

Figure 4. The same as in Figure 3 for the narrowband channel (100 kHz) for June $23(a, c)$ and June $26(b, d), 2013$ 
Discrete cosmic radio sources when moving through the scanned area are passing through particularly oriented $\mathrm{AD}$, and there is a relatively short observation interval for the chosen DCR once a day. Since the maximum AD width is approximately a half of degree, the DCR passes through AD for 15 min. This time interval is sufficient to store DCR ionospheric scintillations in a storage device.

Figures 3 and 4 illustrate a variation in the estimated characteristics of images and a variation in the power of a received signal as the DCR moves for the broadband $(500 \mathrm{kHz})$ and narrowband $(100 \mathrm{kHz})$ channels respectively. The images are plotted for a relatively quiet day without scintillations (June 23, 2013 - Figure 3, a,c; 4, a,c) and for two days with significant scintillations (June 28, 2013 - Figure 3, b, $d$; June 26, 2013 - Figure 4, $b, d$ ). Obviously, there is no considerable difference in the behavior between the calculated parameters under different conditions; hence scintillations have no effect on the size and position of the DCR image. This is due to the fact that the aerial spacing is $5 \mathrm{~m}$. It is much less than the first Fresnel zone for the ionospheric irregularities occurring in this case, therefore we can observe only simultaneous amplitude scintillations in the half-horns.

Since IISR works in the normal mode for about 50-100 days a year, despite the short daily observation period there are sufficiently extensive data for processing. Table lists mean values of some estimated characteristics for summer and winter 2013. It is noteworthy that there is no substantial difference between these characteristics for different seasons.

\begin{tabular}{|l|l|l|}
\hline Season & Summer & Winter \\
\hline Average scintillation index & 0.031 & 0.034 \\
\hline Mean width of DCR image in frequency & 46.642 & 45.875 \\
\hline Mean width of DCR image in phase & 47 & 47.375 \\
\hline $\begin{array}{l}\text { Mean deviation of maximum position } \\
\text { from linear path (in frequency) }\end{array}$ & 14.328 & 18.184 \\
\hline
\end{tabular}

\section{CONCLUSION}

In this paper, we have proposed a method for analyzing the image of the DCR Cygnus-A obtained from statistical distributions of interferometric characteristics of a received signal. In comparison to the analysis of statistical moments, the analysis of the shape of statistical distributions more qualitatively represents DCR characteristics such as position and angular size. We utilized the method to analyze ionospheric scintillations of the DCR Cygnus-A. The DCR ionospheric scintillations registered by IISR were shown to have no effect on parameters of the DCR image, in particular on phase variations. This result is in line with the previously obtained ones [Spoelstra, Yang Yi-Pei, 1995]. Since the aerial spacing is much smaller than sizes of irregularities, phase scintillations can be watched only for super-small-scale irregularities (meters and tens of meters). Observing phase scintillations for large-scale irregularities requires simultaneous observations with distant recorders to enlarge the interferometer base. It has been 


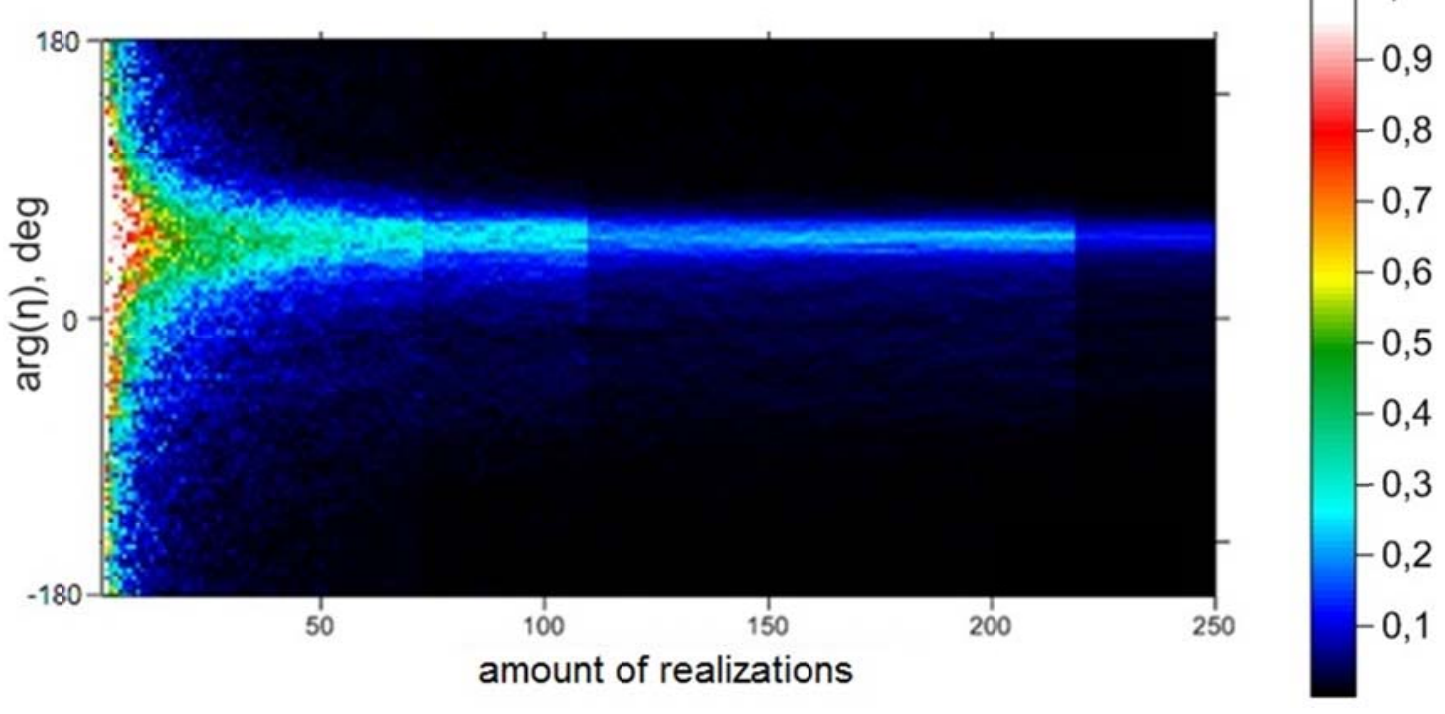

Figure 5. Dependence of the histogram width on the amount of summed realizations for June 22, 2013

demonstrated that there are no substantial differences between statistical parameters in different seasons. It may be due to the fact that signals were recorded with minimum possible integration; therefore we examined in addition the effect of integration time on the size of the source image. Figure 5 illustrates a change in the width of statistical distribution of the correlation coefficient argument depending on a given amount of realizations involved in calculation of the correlation coefficient. It is apparent that as the amount of realizations increases, the width considerably decreases and then becomes virtually constant. It becomes constant after 100 realizations, which corresponds to an integration time of $4 \mathrm{~s}$. It is advised to employ the time for further analysis of data acquired in interferometric studies of the signal from the radio source Cygnus-A.

The statistical characteristics of DCR images, determined using the method described in this work, should thereafter be related to characteristic sizes of ionospheric irregularities as well as to their intensity. This requires simulating the passage of DCR emission through an inhomogeneous ionosphere, utilizing, for example, a phase screen method. Since the study has revealed that the most likely value of statistical distribution and its width are unrelated to radio signal scintillations, works involving simulations must call attention to the shape of distribution itself (asymmetry, granularity) and its variations depending on phase screen characteristics.

The study was made possible by RFBR grant No. 13-05-03946 A.

\section{APPENDIX}

Let a plane wave come to a point at an angle $\theta$ (Figure 1App). Consider signals at the output of two independent antennas with phase centers located at a distance $d$ :

$$
S_{1}=I_{0} e^{i \varphi}+N_{1} e^{i \psi_{1}}
$$




$$
S_{2}=I_{0} e^{i(\varphi+d \sin \theta)}+N_{2} e^{i \psi_{2}}
$$

where $I_{0}$ is an intensity of a source, $N_{1}, N_{2}$ are intensities of noises appearing in reception paths. Phases $\varphi$ and $\psi$ vary from 0 to $2 \pi$ and are independent of each other. Signal power in one of the receivers can be written as follows:

$$
P_{1}=\left\langle S_{1} S_{1}^{*}\right\rangle=\left\langle\left(I_{0} e^{i \varphi}+N_{1} e^{i \psi_{1}}\right)\left(I_{0} e^{-i \varphi}+N_{1} e^{-i \psi_{1}}\right)\right\rangle=\left\langle I_{0}^{2}+I_{0} N_{1} e^{i\left(\varphi-\psi_{1}\right)}+I_{0} N_{1} e^{i\left(\psi_{1}-\varphi\right)}+N_{1}^{2}\right\rangle,
$$

where angle brackets imply averaging. Signal and noise phases being independent, in the averaging the second and third terms of the sum become zero; then Equation (3) is as follows:

$$
P_{1}=\left\langle I_{0}^{2}\right\rangle+\left\langle N_{1}^{2}\right\rangle
$$

For the second receiver, we can write a similar equation

$$
P_{2}=\left\langle I_{0}^{2}\right\rangle+\left\langle N_{2}^{2}\right\rangle
$$

For two signals, we can calculate the mutual correlation coefficient

$$
\eta=\frac{\left\langle S_{1} S_{2}^{*}\right\rangle}{\sqrt{P P_{21}}}=\frac{\left\langle S_{1} S_{2}^{*}\right\rangle}{\left\langle I_{0}^{2}\right\rangle+\left\langle N_{0}^{2}\right\rangle},
$$

where

$$
\left\langle N_{0}^{2}\right\rangle=\left\langle N_{1}^{2}\right\rangle=\left\langle N_{2}^{2}\right\rangle
$$

By substituting (1) and (2) in (6), obtain

$$
\begin{aligned}
& \eta=\frac{\left\langle\left(I_{0} e^{i \varphi}+N_{1} e^{i \psi_{1}}\right)\left(I_{0} e^{-i(\varphi+d \sin \theta)}+N_{2} e^{-i \psi_{2}}\right)\right\rangle}{\left\langle I_{0}^{2}\right\rangle+\left\langle N_{0}^{2}\right\rangle}= \\
& =\frac{\left\langle I_{0}^{2} e^{-i d \sin \theta}+I_{0} N_{1} e^{i\left(\psi_{1}-\varphi-d \sin \theta\right)}+I_{0} N_{2} e^{i\left(\varphi-\psi_{2}\right)}+N_{1} N_{2} e^{i\left(\psi_{1}-\psi_{2}\right)}\right\rangle}{\left\langle I_{0}^{2}\right\rangle+\left\langle N_{0}^{2}\right\rangle} .
\end{aligned}
$$

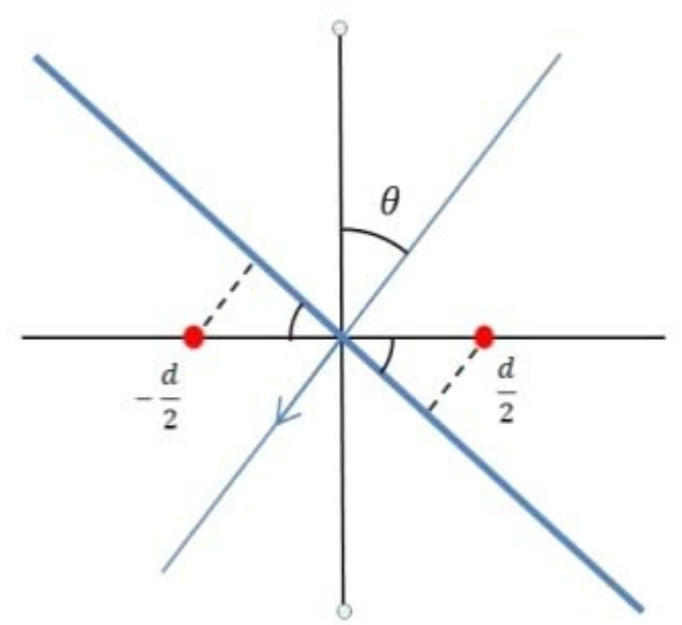

Figure 1App. Geometry of the experiment 
In the numerator of (8), all terms of the sum, except for the first one, in the averaging also become zero due to the independent phases. The equation for the mutual correlation coefficient takes the following form:

$$
\eta=\frac{\left\langle I_{0}^{2} e^{-i d \sin \theta}\right\rangle}{\left\langle I_{0}^{2}\right\rangle+\left\langle N_{0}^{2}\right\rangle}=\frac{\left\langle e^{-i d \sin \theta}\right\rangle}{1+\left\langle N_{0}^{2}\right\rangle /\left\langle I_{0}^{2}\right\rangle} .
$$

This suggests that the modulus of correlation coefficient determines the signal-to-noise ratio

$$
\bmod (\eta)=\frac{1}{1+\left\langle N_{0}^{2}\right\rangle /\left\langle I_{0}^{2}\right\rangle},
$$

and its argument defines the direction of arrival:

$$
\arg (\eta)=-d \sin \theta
$$

\section{REFERENCES}

Bezrodny V.G., Charkina O.V., Yampolsky Yu.M., et al. Research into stimulated ionospheric scintillation and absorption of emission of discrete space sources using panoramic HF riometer. Radiofizika i radioastronomiya [Radio Physics and Radio Astronomy] 2010, vol. 15, no. 2, pp. 151-163 (in Russian).

Kung Chie Yeh, Chao-Han Liu. Radio wave scintillations in the ionosphere. Proc. IEEE. 1982, vol. 70, no. 4, pp. 324-360. DOI: 10.1109/PROC.1982.12313.

Lebedev V.P., Medvedev A.V., Kushnarev D.S. Method of calibration of the directional pattern of the Irkutsk Incoherent Scatter Radar. Proc. of Baikal Young Scientists' International School on Fundamental Physics. IX Young Scientists' Conference "Physical Processes in Outer and Near-Earth Space” XIV Conference "Interaction of Fields and Radiation With Matter'. Irkutsk, 11-16 September 2006. Irkutsk, 2006, pp. 185-188 (in Russian).

Lebedev V.P., Medvedev A.V., Tolstikov M.V. Interfeometric measurements of ionospheric disturbances at the Irkutsk Incoherent Scatter Radar. Vestnik Sibirskogo Gosudarstvennogo Aerokosmicheskogo Universiteta Imeni Akademika M.F. Reshetneva [Bull. of Academic M.F. Reshetnev Siberic State University]. 2013, no. 5 (51), pp. 160163 (in Russian).

Little C.G., Maxwell A. Fluctuations of the intensity of radio waves from galactic sources. Philosophical Magazine Series 7. 1951, vol. 42, iss. 326, pp. 267-278. DOI: 10.1080/ 14786445108561264.

Priyadarshi S. A Review of ionospheric scintillation models. Surv. Geophys. January 2015, vol. 36, pp. 295-324. DOI: $10.1007 /$ s10712-015-9319-1.

Spoelstra T.A.T., Kelder H. Effects produced by the ionosphere on radio interferometry. Radio Sci. 1984, vol. 19, no. 3, pp. 779-788. DOI: 10.1029/RS019i003p00779.

Spoelstra T.A.T., Yang Yi-Pei. Ionospheric scintillation observations with radio interferometry. J. Atm. SolarTerr. Phys. 1995, vol. 57, no. 1, pp. 85-97.

Sukumar S. Ionospheric refraction effects on radio interferometer phase. J. Astrophys. Astr. June 1987, vol. 8, pp. 281-294. DOI: 10.1007/BF02714893.

Vasilyev R.V., Kushnarev D.S., Lebedev V.P., et al. Perspectives of usage of Irkutsk Incoherent Scatter Radar (IISR) as an imaging riometer and radio heliograph. J. Atm. Solar-Terr. Phys. 2013, vol. 105/106, pp. 273-280. DOI: $10.1016 /$ j.jastp.2013.06.012.

The article is based on materials of the XIV th Young Scientists' Conference "Interaction of fields and radiation with matter" of Baikal Young Scientists' International School on Fundamental Physics (September 14-18, 2015, Irkutsk, Russia) 